\section{Exposición a nitratos en agua y su relación con disfunción de la glándula tiroides: revisión sistemática ¿Existen riesgos para la salud de la población?}

\author{
RODRIGO DONOSO M. ${ }^{1,4, a}$, SANDRA CORTÉS A. .,2,3,b
}

\section{Exposure to nitrates in drinking water and its association with thyroid gland dysfunction}

Background: Nitrate exposure may be associated with thyroid gland dysfunction. Aim: To review the available evidence about the relationship between nitrates in drinking water and thyroid gland dysfunction. Material and Methods: A wide search was performed using Medline, Cochrane, Lilacs, IBECS and Scielo databases using pertinent keywords, finding a total of 66 related studies. After filtering and in depth reviewing, a total of 12 studies were included in this review. Results: The main results reveal the importance of this ion for human health, finding evidence both in animals and human beings that suggest pathological changes in the gland as its relationship with the occurrence of subclinical hypothyroidism, and potentially cancer of the thyroid gland. In Chile, nitrate is not considered a critical contaminant so its regular measuring and control is not enforced. Conclusions: In light of the present review we believe that there is evidence to consider nitrate as a critical contaminant whose measurement, registration and correct implementation of valid policies would have a direct benefit for the population of this country. Without this information, it is not possible to quantify the damage to human health, especially in vulnerable groups residing in areas at greatest risk of exposure.

(Rev Med Chile 2018; 146: 223-231)

Key words: Chile; Drinking; Nitrates; Thyroid Gland.

\author{
'Departamento de Salud Pública, \\ Facultad de Medicina, Pontificia \\ Universidad Católica de Chile. \\ Santiago, Chile. \\ ${ }^{2}$ Advanced Center for Chronic \\ Diseases (ACCDis), Pontificia \\ Universidad Católica de Chile. \\ Santiago, Chile. \\ ${ }^{3}$ Centro de Desarrollo Urbano \\ Sustentable CEDEUS. Santiago, \\ Chile. \\ ${ }^{4}$ Facultad de Medicina CAS, \\ Universidad del Desarrollo. \\ Santiago, Chile. \\ `Médico, MSc Epidemiología. \\ ${ }^{\mathrm{b}} \mathrm{MV}, \mathrm{Mg}$ Cs Biológicas, PhD en \\ Salud Pública.
}

Este trabajo no recibió apoyo financiero.

Los autores declaran no tener conflictos de interés.

Recibido el 17 de julio de 2017, aceptado el 29 de agosto de 2017.

Correspondencia a: Sandra Cortés Arancibia Diagonal Paraguay 362 , Departamento de Salud Pública, segundo piso. Santiago, Chile. scortes@med.puc.cl
E 1 nitrato, forma más oxidada del nitrógeno que se encuentra en la naturaleza $\left(\mathrm{NO}^{-}\right)^{1}$, es reconocido mundialmente en la actualidad como un contaminante de las aguas para consumo humano. Las principales fuentes para esta contaminación provienen del uso de fertilizantes agrícolas, pesticidas, residuos animales, y siembras de legumbres que capturan nitrógeno atmosférico depositándolo en la tierra ${ }^{2}$. Estas fuentes de nitrógeno han aumentado de manera exponencial desde los años 50, lo que se ve reflejado en un aumento de compuestos oxidados, como el nitrato en el agua de consumo humano.

Uno de los efectos descritos a cabalidad que inciden directamente sobre la salud humana es la relación entre el nitrato y la metahemoglobinemia en lactantes. Este síndrome, más conocido como el "niño azul", consiste en la formación de una molécula de hemoglobina más oxidada que le otorga una mayor captación de oxígeno, haciendo que su transporte sea menor al no poder entregarlo a los tejidos y provocando la cianosis periférica característica. Esta evidencia determinó que la OMS estableciera en 1971 una recomendación para regular las cantidades máximas de nitrato a permitir en aguas para consumo, ubicándolo en $45 \mathrm{mg} / \mathrm{l}$ para el nitrato, $3 \mathrm{mg} / \mathrm{l}$ para el nitrito y una razón entre ambos niveles y sus límites permitidos 
sumados que no debe exceder el valor $1^{3}$. Esta norma por lo general, de acuerdo a estadísticas de la OMS, se cumple en aguas para consumo urbanas, pero en aguas provenientes de pozos o en áreas rurales este valor puede encontrarse ampliamente excedido. Sin embargo, estudios en Israel muestran que la media del porcentaje de casos de metahemoglobinemia en infantes, independiente de la concentración de nitratos encontrada en aguas para consumo, es aproximadamente de $1,04 \%{ }^{4}$. En Chile no se reportan casos de metahemoglobinemia infantil.

En relación a otras manifestaciones asociadas a este ion, existe creciente evidencia desde los años 70 en torno a la influencia que pudiese tener su exposición con disfunciones de la glándula tiroides. Esta relación se comienza a sospechar debido a hallazgos de bocio eutiroideo en zonas con buena suplementación con iodo ${ }^{5}$. Algunos estudios desde entonces han tratado de explicar esta asociación tanto en modelos animales como en estudios poblacionales.

El propósito del presente estudio es evaluar la evidencia disponible acerca de la exposición a nitratos en agua para consumo humano y su relación con la disfunción de la glándula tiroides, lo que podría contribuir a la implementación o mejora de las políticas existentes en materia de regulación sanitaria para este ion.

\section{Material y Métodos}

Utilizando una estrategia de búsqueda amplia en bases de datos reconocidas por contar con revisión de pares, tales como Medline, Cochrane, Lilacs, IBECS y Scielo acerca de los tópicos de interés: agua (water), nitrato (nitrate) y tiroides (thyroid OR gland OR thyroid gland) se encontraron un total de 66 artículos, sin filtro de años. La revisión por pertinencia del título de cada artículo excluyó un total de 35.

Se establecieron los siguientes criterios de inclusión:

Artículos que evaluaran la relación entre el nitrato en agua y la función de la tiroides.

Artículos en inglés o castellano.

Artículos publicados hasta marzo de 2016.

Artículos disponibles en versión completa en bibliotecas UC.

Se excluyeron estudios que abordaran otras relaciones endocrinas asociadas a la exposición a nitratos y aquellos estudios en otros idiomas que no fueran inglés o español.

El análisis de los abstracts encontrados excluyó un total de 18 artículos. Se realizó una búsqueda por artículos similares con los artículos incluidos encontrándose 5 artículos más que aportan al objetivo de la revisión. En total se evalúan 18 artículos pertinentes. En una última etapa se excluyeron 6 artículos durante la revisión en profundidad debido al no cumplimiento de los criterios de inclusión.

El número de artículos utilizados finalmente para el análisis de esta revisión suma un total de 12 (Figura 1).

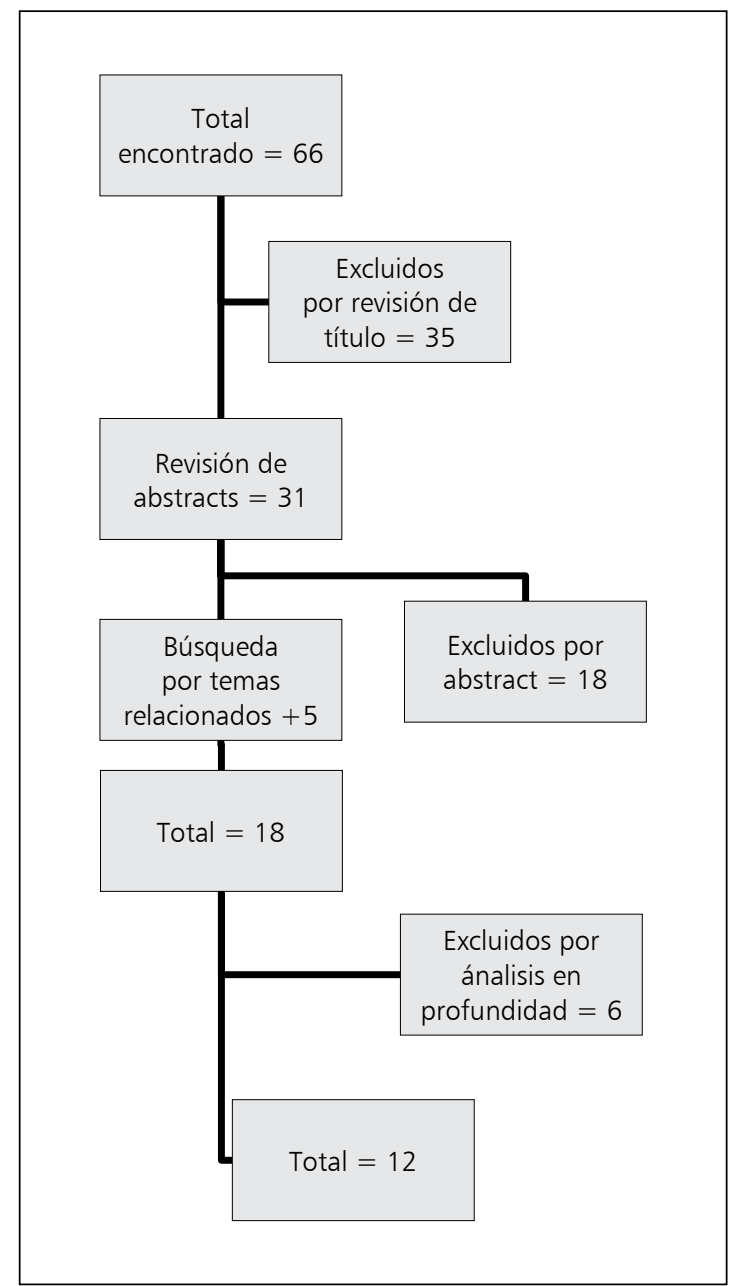

Figura 1. Estrategia de búsqueda ampliada de evidencia de efectos tiroideos y nitratos en agua. 


\section{Resultados}

Entre los estudios revisados que analizan la relación entre el nitrato y la función de la glándula tiroides, destacan estudios experimentales randomizados en animales, específicamente ratas de la especie Wistar y estudios clínicos y observacionales en humanos (Tabla 1).

En animales, la disfunción tiroidea estaría fundamentada en la interferencia que produce el nitrato en la capacidad de captación y acumulación de iodo por parte de la tiroides, ion fundamental para su funcionamiento endocrino. Esta interferencia ocurre por la capacidad del nitrato de competir con el iodo en el transportador sodio-iodo ubicado en la membrana celular tiroidea; dicho transportador capta y fija el iodo de manera activa dentro de los folículos de la glándula 6 . Así, en los modelos animales, se han observado cambios morfológicos importantes que explican el bocio, dentro de los cuales destacan: vacuolización y un aumento del tamaño de los folículos con un aplanamiento del epitelio folicular tiroideo ante exposiciones a concentraciones superiores a 150 $\mathrm{mg} / \mathrm{l}^{7}$. Al comparar marcadores de disfunción tiroidea entre los grupos de ratas expuestos y no expuestos, estos estudios demuestran mayor peso de la tiroides en todos los grupos expuestos, independiente de la concentración de nitrato administrada y disminución de la actividad de la peroxidasa tiroidea (enzima que cataliza la reacción del ion yoduro a yodo para su incorporación a las precursoras de las hormonas tiroideas en la glándula), disminución de T4 total, sin resultados consistentes respecto a T3, observándose concomitantemente un aumento de la TSH y excreción urinaria de yodo, dando cuenta de la menor capacidad de la tiroides de utilizar este ion para sintetizar las hormonas ${ }^{1,7,8}$.

Respecto a estudios en humanos, destaca un sólo estudio clínico randomizado, realizado en los Países Bajos en el año 2007, en el cual se dividen los individuos en 2 grupos de 10 personas, a los cuales se expone a nitrato en altas concentraciones $(15 \mathrm{mg} / \mathrm{kg}$ por 28 días a beber en agua) junto a una dieta restringida en yodo, mientras que, al otro grupo se le entrega un placebo (agua destilada), siguiendo la misma dieta que el grupo experimental. Al inicio del protocolo y un mes posterior a la exposición se mide el promedio de captación de iodo de ambos grupos, no encontrándose di- ferencias significativas ${ }^{9}$, lo que se podría explicar en parte por el largo intervalo de tiempo entre exposición y medición del resultado. También debe considerarse, tal como sugieren otros resultados en esta revisión, que los cambios funcionales y morfológicos no ocurrirían como resultado de una exposición aguda, sino que se observarían como resultado de múltiples exposiciones a nitrato en altas concentraciones a lo largo del tiempo, lo que se consideraría una exposición crónica.

Dentro de los estudios observacionales que buscan develar la relación de una exposición crónica a nitrato con disfunciones de la glándula tiroides, se describen antecedentes desde el año 1994. En un estudio realizado en los Países Bajos se establecieron diferencias significativas en el volumen tiroideo entre el grupo de alta exposición (definido como comunidad con niveles de exposición a nitratos en agua $>17,5 \mathrm{mg} / \mathrm{l}$ ) y los otros dos grupos de exposición baja $(0,02 \mathrm{mg} / \mathrm{l})$ y media $(17,5 \mathrm{mg} / \mathrm{l})$, observándose que a mayores niveles de nitratos en agua se reporta un mayor volumen de la glándula tiroides (en la zona media-baja el volumen es de 8,3 a $8,9 \mathrm{~cm}^{3}$ respectivamente, mientras que en la zona media-alta el volumen varia de 8,2 a $11,1 \mathrm{~cm}^{3}$, siendo la diferencia de este grupo estadísticamente significativa respecto al grupo bajo y medio); además se observa una relación inversa entre el volumen tiroideo y la hormona tiroestimulante (TSH) ${ }^{5}$.

Este estudio sienta las bases para varias líneas de investigación en el tema, dentro de las cuales, una de las más extensas es la de Gatseva et al, grupo que se ha dedicado a analizar, dentro de otras exposiciones, el perclorato y nitrato, principalmente. Entre los trabajos acerca del nitrato, su línea de investigación se ha desarrollado en Bulgaria con publicaciones desde 1998, siendo éstos en base a población infantil y embarazadas. Estudios realizados en 2007, de tipo ecológico, revelan un mayor riesgo de disfunción tiroidea como consecuencia de vivir en comunidades con altas concentraciones de nitratos en agua, determinando la ocurrencia de niveles alterados de yoduria. Estos autores establecieron que en comunidades expuestas a niveles entre 75 a $93 \mathrm{mg} / \mathrm{l}$ de nitratos en el agua se observa valores menores de yoduria al comparar con una comunidad con niveles de $8 \mathrm{mg} / \mathrm{l}$ de nitratos en agua. Al considerar la disfunción tiroidea como una consecuencia de salud, se observó un mayor riesgo de disfunción tiroidea $(\mathrm{OR}=5,29$; IC 95\% 


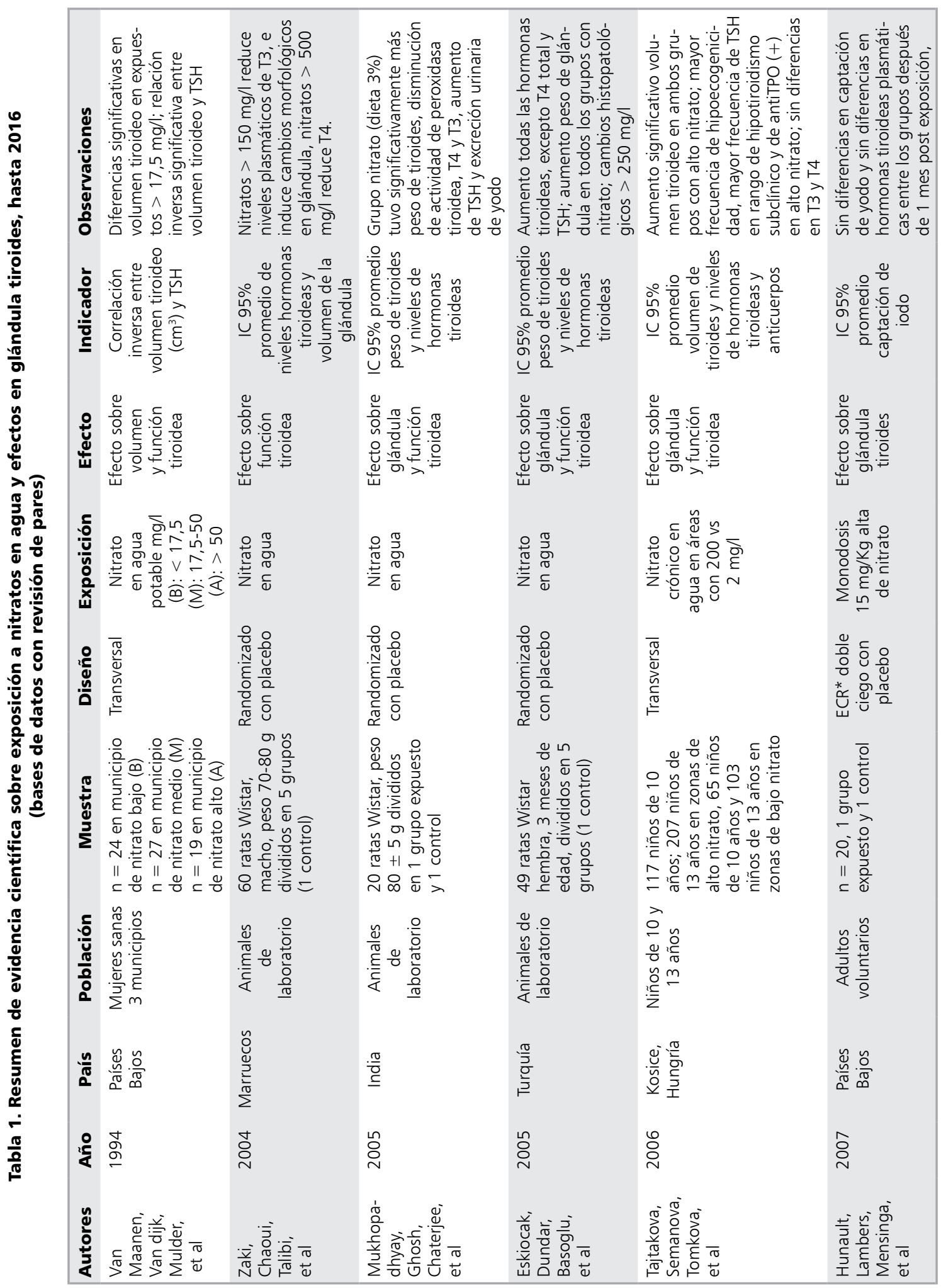




\begin{tabular}{|c|c|c|c|c|c|}
\hline 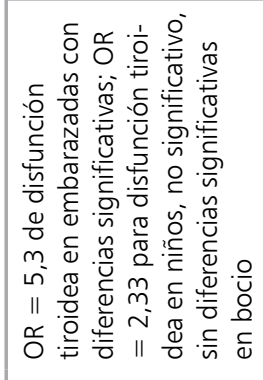 & 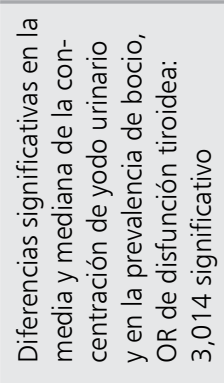 & 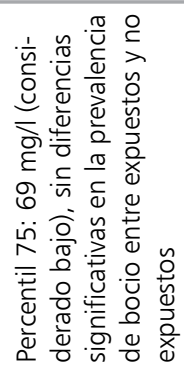 & 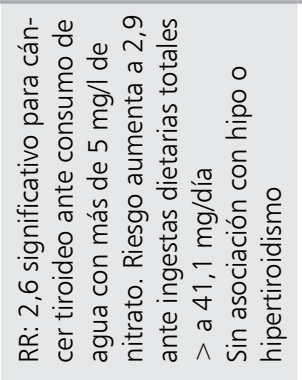 & 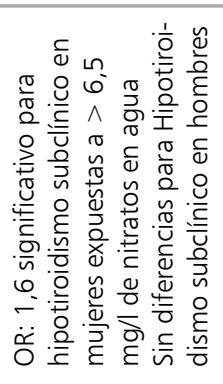 & 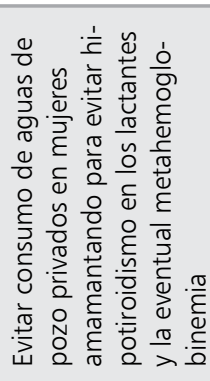 \\
\hline$\stackrel{\circ}{0}$ & 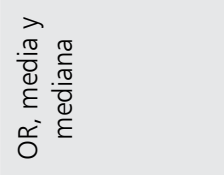 & $\begin{array}{l}\stackrel{\circ}{\text { in }} \\
\text { u }\end{array}$ & $\stackrel{\propto}{\propto}$ & 응 & \\
\hline 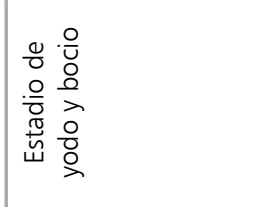 & 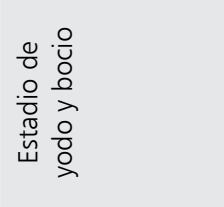 & 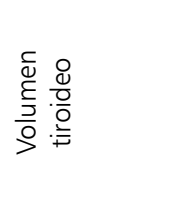 & 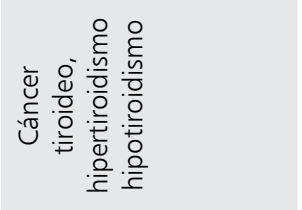 & 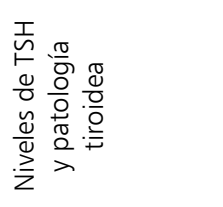 & \\
\hline 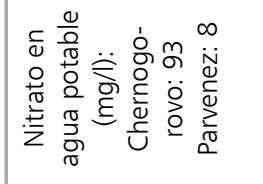 & 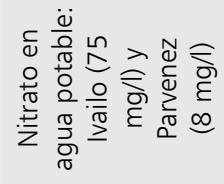 & 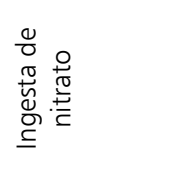 & 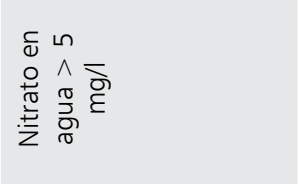 & 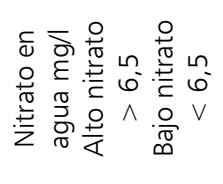 & \\
\hline $\begin{array}{l}\frac{8}{0} \\
\frac{0}{0} \\
\text { ư }\end{array}$ & 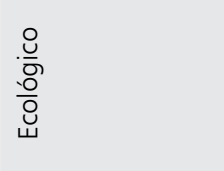 & 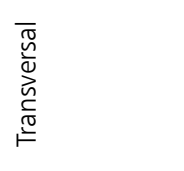 & $\begin{array}{l}\frac{y}{0} \\
\frac{0}{0} \\
\frac{0}{0}\end{array}$ & 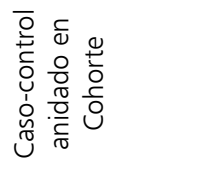 & 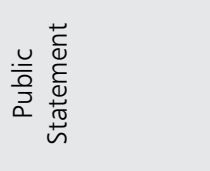 \\
\hline 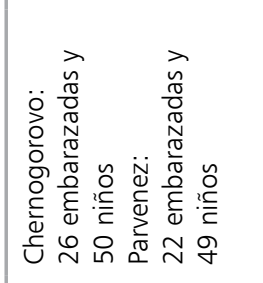 & 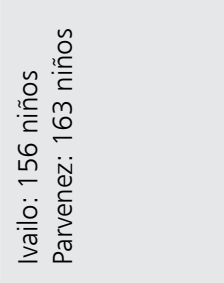 & 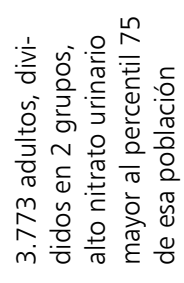 & 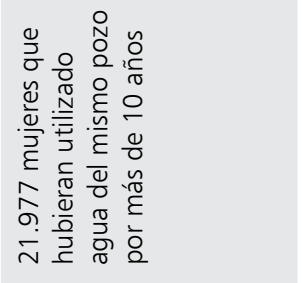 & 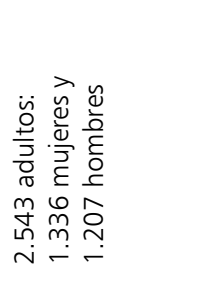 & \\
\hline 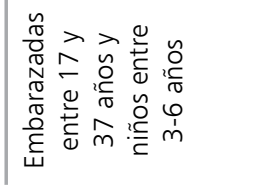 & 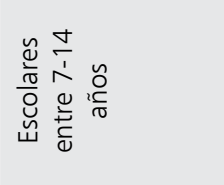 & 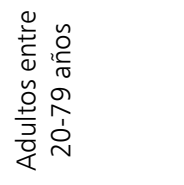 & 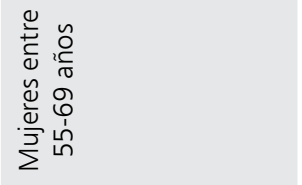 & 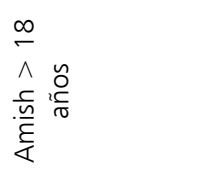 & \\
\hline $\begin{array}{l}\frac{0}{\frac{0}{5}} \\
\frac{0}{5} \\
\frac{0}{5}\end{array}$ & $\begin{array}{l}\frac{0}{\frac{0}{5}} \\
\frac{\sqrt{5}}{3} \\
\frac{0}{12}\end{array}$ & 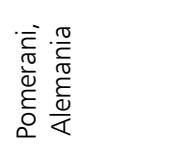 & 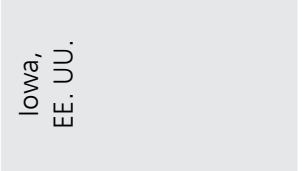 & 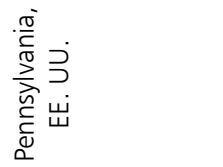 & $\begin{array}{l}\grave{3} \\
\text { نं }\end{array}$ \\
\hline 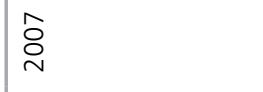 & ঠे & 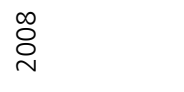 & $\stackrel{\circ}{\stackrel{\sim}{2}}$ & $\stackrel{\sim}{\sim}$ & $\stackrel{+}{\sim}$ \\
\hline 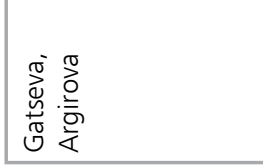 & 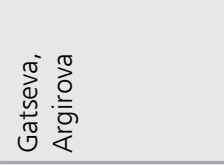 & 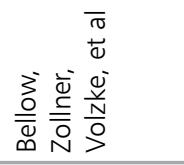 & 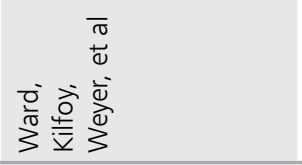 & 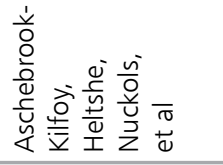 & 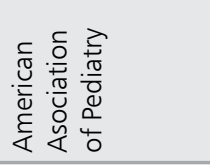 \\
\hline
\end{tabular}


[1,003-27,94]) en las mujeres embarazadas; no se establece mayor riesgo de estas patologías en población infantil entre 3-6 años (OR 2,33; IC $95 \%[0,85-6,41])$. Al mirar la misma relación en escolares entre 7-14 años se observa un riesgo significativo de disfunción tiroidea en los pueblos de alta concentración de nitrato $(75 \mathrm{mg} / \mathrm{l})$ con un $\mathrm{OR}=3,01$ (IC 95\% [1,293-7,027]).

Así mismo, un estudio en Hungría con niños entre 10 y 13 años compara 4 grupos de niños, un grupo de 117 niños de 10 años y otro de 207 niños de 13 años que viven en zonas de alta exposición a nitrato (51-274 mg/l) y otros 2 grupos, uno de 65 niños de 10 años y otro de 103 niños de 13 años, en zonas de baja exposición a nitrato $(<2 \mathrm{mg} / \mathrm{l})$, a fin de evaluar el efecto de esta exposición sobre la tiroides. Este estudio encuentra diferencias significativas en el volumen tiroideo, observándose mayor volumen promedio en los grupos con alta exposición a nitrato: $5,1 \pm 0,14 \mathrm{ml} / \mathrm{m}^{2}$ para el grupo etario de 10 años y $5,97 \pm 0,11 \mathrm{ml} / \mathrm{m}^{2}$ para el grupo de 13 años, además de una mayor frecuencia de hipoecogenicidad de la glándula $(13,7 \%$ en grupos con alta exposición versus $4,7 \%$ en grupos con baja exposición $(\mathrm{p}<0,01)$ para los grupos de 10 años y $10,6 \%$ versus $5,7 \%(\mathrm{p}<0,03)$ entre los grupos de 13 años). Estos autores establecieron el volumen tiroideo con ultrasonido y aplicaron una fórmula validada por otros autores que expresan el volumen como $\mathrm{ml} / \mathrm{m}^{2}$ de superficie corporal. Al analizar las hormonas tiroideas séricas, observaron mayor frecuencia de TSH elevadas sin diferencias en T3 y T4, lo que estaría en el contexto de una mayor frecuencia de hipotiroidismo subclínico $(4 \%)$ y una mayor frecuencia de antiTPO $(+)(2,5 \%)$ en los grupos expuestos a altos niveles de nitrato, comparado con $0 \%$ para ambas mediciones en el grupo de baja exposición; los autores señalan que aun cuando ellos no midieron nitratos en orina, se reportan en el área en estudio otras actividades que podrían aumentar la ingesta de nitratos por otras vías que también usan agua enriquecida por nitratos, por ejemplo, cocinar, riego de sus propios vegetales o para alimentar animales; sin embargo, también se requiere considerar otros factores hereditarios $\mathrm{s}^{9,10,11,12,13}$.

Por otra parte, estudios en Alemania en 2008, realizados de manera transversal con una muestra de 3.773 adultos entre 20-79 años en que se comparan dos grupos dentro de la misma población en base a la concentración de nitrato urinario ${ }^{12}$ define el grupo expuesto como aquellos que superan el percentil 75 de los valores de la excreción de nitrato en orina ( $>69 \mathrm{mg} / \mathrm{l}$ para esta población), no logrando demostrar diferencias significativas en la prevalencia de bocio entre el grupo considerado expuesto y el no expuesto.

Sin embargo, estudios de cohorte realizados en Estados Unidos de América (EE. UU.), revelan importantes hallazgos que sugieren una relación más plausible entre el nitrato y la patología tiroidea, aun cuando no se establece totalmente una causa-efecto ${ }^{2,15}$. En una cohorte retrospectiva de mujeres entre 55-69 años en el estado de Iowa en 2010 revela un riesgo relativo (RR) igual a 2,6 (IC 95\% [1,1-6,2]) para cáncer tiroideo en mujeres con consumo superior a $5 \mathrm{mg} / \mathrm{l}$ de nitrato en el agua, aun cuando no logra demostrar una asociación con hipo o hipertiroidismo. Al realizar un análisis más detallado de la cohorte comparando el cuartil inferior de ingesta total de nitratos $(<17,4$ $\mathrm{mg} /$ día) con el cuartil superior (>41,1 mg/día) se observa un $\mathrm{RR}=2,9$ (IC 95\% [1,0-8,1], p = 0,046) para cáncer y un OR = 1,2 (IC 95\% [1,1-1,4]) para hipotiroidismo, sin encontrar una asociación significativa para la prevalencia de hipertiroidismo ${ }^{2}$. Otro estudio caso y control dentro de una cohorte de 2.543 Amish mayores de 18 años para evaluar los niveles de TSH y patología tiroidea debido a la exposición a nitrato, estableció un riesgo de (OR $=1,6$; IC 95\% [1,11-2,32]) para hipotiroidismo subclínico sólo en mujeres, no así en hombres o para hipotiroidismo clínico en ambos sexos ${ }^{15}$.

Con esta evidencia la Academia Americana de Pediatría decide emitir en 2014 su comunicado al respecto, recomendando evitar consumo de aguas de pozo privados por parte de mujeres amamantando para evitar tanto hipotiroidismo subclínico en los lactantes o eventualmente la relación previamente conocida y poco frecuente con la metahemoglobinemia ${ }^{6}$.

En nuestro país (Chile), la norma de aguas para uso humano para el nitrato está fijada acorde con la recomendación propuesta por la OMS; pero está decretado como un contaminante "no crítico"16 por lo que sus valores en agua potable son mayoritariamente desconocidos. En la VIII región se llevó a cabo un estudio en 2012 para determinar estos niveles, comparando mediciones de nitrato en aguas rurales utilizando como control el agua potable de la ciudad de Concepción, encontrándose un promedio de $23,29 \mathrm{mg} / \mathrm{l}$ de nitrato en aguas 
Tabla 2. Población expuesta a nitratos en agua con concentraciones mayores a $50 \mathrm{mg} / \mathrm{l}$ durante meses de excedencia el año 2015 en Chile

\begin{tabular}{|clcc|}
\hline Región & Servicio & $\begin{array}{c}\text { Concentración promedio meses } \\
\mathbf{5 0} \mathbf{~ m g / l}\end{array}$ & $\begin{array}{c}\text { Estimación población comunal } \\
\mathbf{2 0 1 5}\end{array}$ \\
\hline III & Chañaral & 64,8 & 13.725 \\
III & Copiapó & 51 & 172.231 \\
\hline III & El Salado Inca de Oro & 72 & 15.224 \\
\hline RM & Izarra & 53,7 & $795^{*}$ \\
\hline V & La Calera & 55 & 55.121 \\
V & Quillota & 61,3 & 93.633 \\
V & San Isidro & 52 & 93.633 \\
\hline
\end{tabular}

Fuente: elaboración propia en base a datos SISS (2016). *Valor estimado a partir de datos de cobertura de empresa sanitaria.

rurales comparado con $3,6 \mathrm{mg} / \mathrm{l}$ en aguas urbanas $^{17}$. Estos resultados muestran que, sólo en este estudio, a pesar, de encontrarse diferencias en el promedio de la concentración de nitratos en aguas para consumo humano entre sectores rurales y urbanos éstos no superan la norma permitida. Sin embargo, los datos de la Superintendencia de Servicios Sanitarios, quien provee información solo para las ciudades que tienen empresas sanitarias en su supervisión, para el año 2015 muestran que, de 926 registros de niveles de nitratos informados por las empresas sanitarias, 117 tienen un valor "0", cumpliendo norma (12,6\%); por otra parte, hay 779 registros $(84,1 \%)$ con valores entre 1 y $49 \mathrm{mg} / \mathrm{l}$ y finalmente hay 30 registros $(3,3 \%)$ con valores mayores de $50 \mathrm{mg} / \mathrm{l}$. En la Tabla 2 se observa aquellos servicios que abastecieron aguas con niveles mayores a lo aceptado por la norma y un valor estimado de la población expuesta a niveles de excedencia (SISS, 2016). No se dispone de información respecto a los sistemas rurales de provisión de aguas para uso humano.

\section{Discusión}

Según evidencia recabada, se puede observar que se han demostrado algunas asociaciones significativas con disfunción de la glándula tiroidea, pero estos son mayoritariamente con disfunciones del tipo subclínico o con bocio eutiroideo, observándose así una falta de evidencia que aborde estos trastornos con manifestaciones clínicas.

Cabe destacar la importante asociación en- contrada por las cohortes americanas, en que destaca un alto riesgo para cáncer de tiroides ante exposiciones tan bajas de nitratos en aguas como $5 \mathrm{mg} / \mathrm{l}$, cifras que pueden ser observadas en aguas para consumo humano en cualquier ciudad de Chile; considerando la reciente evidencia, se requiere más investigación para poder emitir una conclusión decisiva a nivel nacional e internacional. Se debe considerar que muchos de los estudios sólo se enfocan en la concentración de nitrato ingerida en agua, sin controlar la ingesta que pueda ocurrir por otras vías de exposición, como lo es la dieta, aun así, se concluye que el nitrato efectivamente puede representar un riesgo para la salud humana incluso a concentraciones muy bajas, demostrándose en otras especies animales que una exposición crónica causa cambios anatomo-patológicos de la glándula tiroides, y por ende, se deberían realizar mayores esfuerzos para su correcta regulación y monitorización. Es de importancia de salud pública el control de éste y otros contaminantes ambientales que reducen la producción de hormonas tiroideas, dada la relación de éstas con el neurodesarrollo en población infantil (Haddow et al. 1999; Pop et al. 2003, citados por Steinmaus et al, 2016).

Debe considerarse que en nuestro país no es considerado un contaminante de alta relevancia, pero de acuerdo a lo recabado en la literatura internacional debiera ser sujeto de revisión el valor normado a partir de información científica generada en el país que permita establecer sus potenciales daños, incluyendo sus efectos cancerígenos no evaluados en el país. En Chile 
la prevalencia de hipotiroidismo alcanza 19,4\% de acuerdo a datos de la Encuesta Nacional de Salud 2009-2010, observándose mayor frecuencia de esta patología en el sexo femenino y con una distribución espacial en las diferentes regiones del país que fluctúa entre $12,3 \%$ en la X Región a $37,0 \%$ en la Región de Biobío ${ }^{18}$. Estas cifras persistentemente elevadas a pesar de los esfuerzos por ampliar la cobertura de tratamiento y una buena suplementación de yodo en la sal a nivel nacional podrían explicarse por exposiciones a otros agentes ambientales que interfieren con el funcionamiento adecuado de la glándula, tal como ocurre con el nitrato. Chile es un país con alta variabilidad meteorológica que se expresa, entre otras, con incrementos de áreas con sequía, por lo que se espera que estas concentraciones se incrementen, aumentando así los riesgos para las personas expuestas.

\section{Conclusión}

Esta revisión de la literatura logra demostrar la relevancia que tiene el nitrato como contaminante en aguas para consumo humano, y propone, en base a la evidencia existente, un cambio en la importancia que se le otorga a este contaminante. Aún con las limitaciones que puede presentar esta revisión, basada principalmente en la evidencia toxicológica y epidemiológica generada en el extranjero y tomando en cuenta los datos parciales de niveles de exposición disponibles para las aguas de uso humano en las grandes ciudades, supervisadas por la SISS, creemos que existe suficiente evidencia como para establecer sistemas de vigilancia que generen evidencia para nuevos estudios; a la vez parece pertinente mejorar su medición, registro y adecuada fiscalización en pos de un directo beneficio de la población. Más estudios podrán ayudar a revelar las relaciones de este ion con enfermedades de interés como el hipotiroidismo en áreas de mayor riesgo.

Agradecimientos: Los autores quisieran agradecer a la profesora Ana María Sancha por su apoyo y comentarios que ayudaron a la realización de este estudio. Especiales agradecimientos a Proyecto Conicyt/Fondap 15110020. Este trabajo corresponde a una evaluación parte del curso
"Epidemiología Ambiental y Ocupacional”, del Magíster de Epidemiología UC.

\section{Referencias}

1. Mukhopadhyay S, Ghosh D, Chatterjee A, Sinha S, Tripathy S, Chandra AK. Evaluation of possible goitrogenic and antithyroidal effect of nitrate, a potential environmental pollutant. Indian J Physiol Pharmacol 2005; 49 (3): 284-8.

2. Ward MH, Kilfoy BA, Weyer PJ, Anderson K, Folsom AR, Cerhan JR. Nitrate intake and the risk of thyroid cancer and thyroid disease. Epidemiology 2010; 21 (3): 389-95.

3. World Health Organization (US). Guidelines for drinking-water quality: incorporating 1st and 2nd edd, vol.1, Recommendations [Internet]. 3rd ed. Geneva: World Health Organization; 2008. Capítulo 12, Microbial fact sheets; p. 296-460.

4. Shuval HI, Gruener N. Epidemiological and toxicological aspects of nitrates and nitrites in the environment. American journal of public health 1972; 62 (8): 1045-52.

5. Van Maanen JM, van Dijk A, Mulder K, de Baets MH, Menheere PC, van der Heide D, et al. Consumption of drinking water with high nitrate levels causes hypertrophy of the thyroid. Toxicol Lett 1994; 72 (1-3): 365-74.

6. American Academy of Pediatrics. Iodine deficiency, pollutant chemicals, and the thyroid: new information on an old problem. Pediatrics 2014; 133 (6): 1163-6.

7. Zaki A, Chaoui AA, Talibi A, Derouiche AF, Aboussaouira T, Zarrouck K, et al. Impact of nitrate intake in drinking water on the thyroid gland activity in male rat. Toxicol Lett 2004; 147 (1): 27-33.

8. Eskiocak S, Dundar C, Basoglu T, Altaner S. The effects of taking chronic nitrate by drinking water on thyroid functions and morphology. Clin Exp Med 2005; 5 (2): 66-71.

9. Hunault CC, Lambers AC, MensingaTT, van Isselt JW, Koppeschaar HPF, Meulenbelt J. Effects of sub-chronic nitrate exposure on the thyroidal function in humans. Toxico Lett 2007; 175 (1): 64-70.

10. Gatseva P, Lazarova A, Maximova S, Pavlova K. Experimental data on the effect of nitrates entering the organism with the drinking water. Folia Medica 1996; 38 (1): 75-83.

11. Gatseva PD, Argirova MD. High-nitrate levels in drinking water may be a risk factor for thyroid dysfunction in children and pregnant women living in rural Bulgarian areas. Int Hyg Environ Health 2008; 211 (5): 555-9.

12. Gatseva PD, Argirova MD. Iodine status and goitre 
prevalence in nitrate-exposed schoolchildren living in rural Bulgaria. Public health 2008; 122 (5): 458-61.

13. Tajtáková $M$, Semanová Z, Tomková Z, Szökeová E, Majoroš J, Rádiková Ž, et al. Increased thyroid volume and frequency of thyroid disorders signs in schoolchildren from nitrate polluted area. Chemosphere 2006; 62 (4): 559-64.

14. Below H, Zöllner H, Völzke H, Kramer A. Evaluation of nitrate influence on thyroid volume of adults in a previously iodine-deficient area. Int J Hyg Environ Health 2008; 211 (1): 186-91.

15. Aschebrook-Kilfoy B, Heltshe SL, Nuckols JR, Sabra $\mathrm{MM}$, Shuldiner, AR, Mitchell BD, et al. Modeled nitrate levels in well water supplies and prevalence of abnormal thyroid conditions among the Old Order Amish in Pennsylvania. Environ Health 2012; 11 (6). Disponible en: http://www.ehjournal.net/content/11/1/6 [Consultado el 05 de octubre de 2015].

16. Instituto Nacional de Normalización (CL). Nch 409/1. Of. 2005: Agua potable. Santiago: INN; 2005. Disponible en: http://www.mma.gob.cl/1304/articles-52016_Capitulo_5.pdf [Consultado el 05 de octubre de 2015].

17. Schmidt T, Sedaghat S, Rosel P, Fierro J. Medición de nitratos y nitritos en agua y saliva de población rural precordillerana de la VIII región. Rev Otorrinolaringol Cir Cabeza Cuello 2012; 72 (2): 119-24.

18. Ministerio de Salud (CL). Encuesta Nacional de Salud ENS Chile 2009-2010 [Internet]. Disponible: http:// web.minsal.cl/portal/url/item/bcb03d7bc28b64dfe040010165012d23.pdf [Consultado el 29 de junio de 2015].

19. Superintendencia de Servicios Sanitarios (SISS). Resultados de control de calidad del agua. Disponible en: http:// www.siss.cl/577/w3-propertyvalue-3525.html 